\title{
Recent HIV Risk Behavior and Partnership Type Predict HIV Pre-Exposure Prophylaxis Adherence in Men Who Have Sex with Men
}

\author{
Jill Blumenthal, MD, ${ }^{1}$ David J. Moore, MD, MAS, PhD, ${ }^{1}$ Sonia Jain, $\mathrm{PhD},{ }^{1}$ Xiaoying Sun, \\ Eric Ellorin, MAS, ${ }^{1}$ Katya Corado, MD, ${ }^{2}$ Martin Hoenigl, MD, ${ }^{1}$ Michael Dube, MD, ${ }^{3}$ \\ Richard Haubrich, MD, ${ }^{4}$ and Sheldon R. Morris, MD, MPH, \\ for the California Collaborative Treatment Group (CCTG) TAPIR Study Team
}

\begin{abstract}
Men who have sex with men (MSM) reporting higher HIV risk behavior over time are often more adherent to pre-exposure prophylaxis (PrEP), but it is unclear if recent risk behavior and partnership type affect long-term PrEP adherence. HIV-negative MSM and transgender women completing the 48-week randomized study TAPIR (Daily Text Messages to Support Adherence to PrEP in At-Risk for HIV Individuals) were included. At baseline and weeks 24 and 48, a modified Calculated Risk (mCalcR) Score estimated the likelihood of HIV seroconversion over 1 year based on reported condomless anal sex acts in the last month and current sexually transmitted infection. mCalcR scores were categorized as low, moderate, and high/very high risk. Partnership type was classified as no partner/single HIV-negative partner (no/single-), single HIV-positive partner (single+), or multiple partners of any serostatus (multi) in the past 3 months. PrEP adherence was measured by intracellular tenofovir-diphosphate (TFV-DP) levels. Among 313 individuals, there was no difference in $\mathrm{mCalcR}$ category from baseline to week 48 . There was a significant change in partnership type, with no/single partnerships increasing from $0.5 \%$ to $9 \%$. Participants with moderate and high/very risk had higher TFV-DP levels than the low-risk group. No/single participants had lower TFV-DP levels than those reporting single+ or multi. Although there was a shift toward lower-risk partnerships, HIV risk category remained stable over time. Individuals with riskier behaviors and partnerships had higher PrEP drug levels, suggesting continued motivation for and adherence to PrEP.
\end{abstract}

Keywords: HIV prevention, pre-exposure prophylaxis (PrEP), HIV risk, partnerships, men who have sex with men (MSM), medication adherence

\section{Introduction}

$\mathbf{T}$ HREE DECADES AFTER the onset of the AIDS epidemic, men who have sex with men (MSM) continue to bear a disproportionate burden of HIV and account for $67 \%$ of incident infections in the United States. ${ }^{1}$ Promising data in the last several years suggest that taking the two-drug antiretroviral tenofovir disoproxil fumarate/emtricitabine (TDF/FTC) once daily as pre-exposure prophylaxis (PrEP) can greatly reduce the risk of HIV infection in populations at risk for HIV acquisition, including MSM. ${ }^{2-5}$ HIV-negative MSM who engage in high-risk behavior are a priority population for PrEP implementation.

Individual HIV risk is not constant over time. Previous studies suggest that there may be "seasons of risk" in which an individual engages in risk behaviors that may place them at a greater risk of HIV infection than other times. ${ }^{6}$ Similarly, both number of partners and serostatus of partners (i.e., partnership type) change over time and may be a way of conceptualizing HIV risk. For instance, individuals with one single HIV-negative partner are objectively at lower risk for HIV acquisition compared with those in

Departments of ${ }^{1}$ Medicine, ${ }^{2}$ Psychiatry, ${ }^{3}$ Family Medicine and Public Health, University of California San Diego, La Jolla, California.

${ }_{5}^{4}$ Department of Medicine, Los Angeles Biomedical Research Institute at Harbor UCLA Medical Center, Torrance, California.

${ }^{5}$ Department of Medicine, Keck School of Medicine at the University of Southern California, Los Angeles, California.

${ }^{6}$ Gilead Sciences, Inc., Foster City, California.

Original data of this article have been presented in part at IDSA 2017 in San Diego, California. 
serodiscordant partnerships or those who have multiple partners.

High-risk HIV acquisition behaviors have been shown to be associated with PrEP adherence. ${ }^{7-9}$ In the PrEP Demo Project, individuals reporting greater exposure to HIV riskhaving two or more condomless anal sex partners in the past 3 months-were more likely to have protective tenofovirdiphosphate (TFV-DP) concentrations in dried blood spots (DBS) than those with fewer partners. ${ }^{7}$ One can hypothesize that individuals in HIV-serodiscordant partnerships may have the highest PrEP adherence due to both knowing that they might be repeatedly exposed to HIV as well as the opportunity to link his or her PrEP-taking behavior to the ART adherence behavior of an HIV-positive partner. The association between partnership type itself and adherence to PrEP has not been specifically evaluated and may be a simple and informative way to predict PrEP adherence.

\section{Methods}

\section{Study setting}

For this analysis, we employed a well-characterized highrisk cohort of MSM and transgender women (TGW) who participated in the California Collaborative Treatment Group (CCTG) 595 TAPIR study (Daily Text Messages to Support Adherence to PrEP in At-Risk for HIV Individuals, a multicenter randomized study; NCT01761643). ${ }^{10}$ The study was conducted from February 2013 to February 2016, and participants were enrolled at four southern California medical centers (University of California, San Diego [UCSD]; University of Southern California; LA Biomed at HarborUniversity of California Los Angeles; and Long Beach Department of Health and Human Services) as part of the CCTG clinical research network. Over a 48-week study period, all participants received once-daily TDF/FTC PrEP in combination with lab safety monitoring, HIV and sexually transmitted infection (STI) testing, and adherence and risk reduction counseling.

TAPIR participants were MSM and TGW, aged 18 or older, and HIV-negative as confirmed by an antigen/antibody $(\mathrm{Ag} / \mathrm{Ab})$ assay or $\mathrm{Ab}$ assay plus HIV nucleic acid test. Additional eligibility criteria included acceptable laboratory values in the past 30 days, no active hepatitis B, and having a persistent elevated risk of HIV acquisition as determined by having one or more of the following criteria: (1) at least one HIV-positive sexual partner for $\geq 4$ weeks; (2) condomless anal intercourse (CAI) with $\geq 3$ male partners who are HIVpositive or of unknown HIV status in the last 3 months; or (3) CAI with $\geq 1$ male partner and STI diagnosis in the last 3 months. The parent study evaluated the effect of a textmessaging intervention (Individualized Texting for Adherence Building, iTAB) on PrEP adherence compared with standard of care. iTAB participants were more likely to have DBS levels corresponding to perfect adherence (seven doses per week). ${ }^{10}$ Data were collected by both confidential inperson interviews and computer-assisted self-interview instruments, which included a survey evaluating various sexual behaviors that increase the risk of HIV acquisition. Study visits occurred at baseline, weeks $4,12,24,36$, and 48 . The current study included only TAPIR participants who completed the week 48 visit including a DBS sample available for drug-level analysis.

\section{Measures}

An objective Calculated Risk (CalcR) Score was developed at UCSD as an alternative tool to evaluate HIV risk based on participant-specific HIV transmission events. The score is a mathematical equation that focuses on sexual transmission methods and biological factors that may increase HIV acquisition: condomless receptive and insertive anal intercourse acts and STIs including gonorrhea, chlamydia, syphilis, and herpes. ${ }^{11}$ The previously used CalcR Score $^{12}$ included shared needle events through intravenous drug use, but these items were removed from the present study due to extremely low reporting of these events $(<3 \%)$. This modified CalcR (mCalcR) Score incorporates event frequencies over the last month and establishes event probabilities of HIV transmission due to CAI acts ${ }^{13}$ and STI occurrence. ${ }^{14}$ The CalcR Score is extrapolated to the percent likelihood of HIV seroconversion in 1 year if sexual activities persisted at the same rate and is categorized into low $(<0.12 \%)$, moderate $(0.12-0.59 \%)$, high $(0.6-5.9 \%)$, and very high $(>5.9 \%)$ risk groups. Classification is based on the average percent likelihood of HIV seroconversion in MSM in the United States. ${ }^{15}$ STI screening assessments were done at baseline and initially every 12 weeks but later changed to 24week intervals. Testing included syphilis (serum RPR and, if positive, confirmatory treponemal test) as well as nucleic acid amplification testing of urine and swabs of pharynx and rectum for chlamydia and gonorrhea (Hologic Aptima). Since STI data were incomplete at weeks 12 and 36, the risk score was only calculated at baseline and weeks 24 and 48 . Less than $2 \%$ of participants were found to be very high risk by $\mathrm{mCalcR}$ Score. As a result, high and very high risk categories were combined. Additional details on mCalcR are provided in Fig. 1.

Partnership type was available based on self-report from the sexual risk survey collected at baseline, weeks 24 and 48 and assigned as no partner/single HIV-negative partner (no/single-), single HIV+ partner (single+), or multiple (multi) partners of any serostatus.

PrEP adherence was measured by intracellular TFV-DP levels from whole-blood DBS using a liquid chromatographytandem mass spectrometry assay previously validated in PK modeling studies for the determination of TFV and FTC in human plasma. ${ }^{16}$ The concentration of TFV-DP in DBS was evaluated as a continuous measure in fmol per punch at weeks 12 and 48. Adherence thresholds are based on PrEP dosing where four doses per week is considered protective and seven doses per week perfect, defined as the lower interquartile value of protective $(719 \mathrm{fmol} / \mathrm{punch})$ and perfect

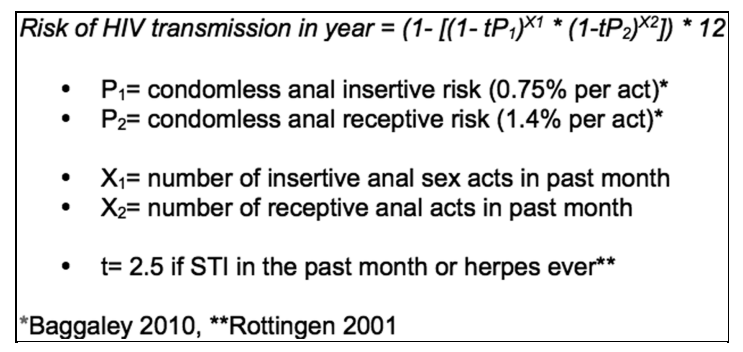

FIG. 1. mCalcR Score formula. mCalcR, modified Calculated Risk. 
Table 1. Demographics and Baseline Risk Factors

\begin{tabular}{|c|c|}
\hline Variable & $\begin{array}{c}\mathrm{n}=313 \\
(\%)\end{array}$ \\
\hline \multicolumn{2}{|l|}{ Gender, $n(\%)$} \\
\hline Men & $310(99)$ \\
\hline Transgender women & $3(1)$ \\
\hline Age, mean (SD) & $35(9)$ \\
\hline \multicolumn{2}{|l|}{ Race, $n(\%)$} \\
\hline Asian & $9(3)$ \\
\hline Black & $36(12)$ \\
\hline White & $240(78)$ \\
\hline Multiple/other & $22(7)$ \\
\hline Hispanic ethnicity & 89 (29) \\
\hline \multicolumn{2}{|l|}{ Education, $n(\%)$} \\
\hline High school or less & $18(6)$ \\
\hline Some college & $118(38)$ \\
\hline Bachelor's degree & $111(36)$ \\
\hline Some graduate or advanced degree & $66(21)$ \\
\hline \multicolumn{2}{|l|}{ Household income, $n(\%)$} \\
\hline$<\$ 2000 /$ month & $65(21)$ \\
\hline$>\$ 2000 /$ month & $202(65)$ \\
\hline Refuse & $46(15)$ \\
\hline \multicolumn{2}{|l|}{ Baseline HIV risk factors $^{\mathrm{a}}, n(\%)$} \\
\hline$\geq 1$ HIV-positive partner for $\geq 4$ weeks & $144(46)$ \\
\hline $\begin{array}{l}\text { CAI with } \geq 3 \text { HIV-positive partners or } \\
\text { unknown in } 3 \text { months }\end{array}$ & $225(72)$ \\
\hline CAI with $\geq 1$ partner and STI in 3 months & $56(18)$ \\
\hline
\end{tabular}

${ }^{a}$ Baseline HIV risk factors are based on study criteria where participants could have more than one baseline HIV risk factor.

CAI, condomless anal intercourse; STI, sexually transmitted infection.

(1246 fmol/punch) levels of TFV-DP derived from the above PK modeling studies. ${ }^{16}$

\section{Statistical analysis}

McNemar's test was used to evaluate change in mCalcR risk category and partnership type from baseline to weeks 24 and 48. Cross-sectional analyses were performed at weeks 12 and 48 to assess the associations between mCalcR risk category and partnership type with adherence. For the week 12 analysis, baseline risk and partnership data were analyzed with week 12 drug levels; for the week 48 analysis, week 48 risk and partnership data were analyzed with week 48 drug levels. Kruskal Wallis and Wilcoxon rank-sum tests were used to compare TFV-DP levels among risk categories and partnership types. Statistical analyses were performed with $\mathrm{R}$ (http://cran.r-project.org), version 3.3.2.

\section{Results}

\section{Study population and demographics}

Of the 313 participants who completed the study, 310 were MSM and 3 were TGW. Median age was 35 with standard deviation of 9 years. The majority of participants were white (78\%), with $12 \%$ black and $28 \%$ Hispanic. More than half of the participants held a bachelor's or advanced degree, and nearly two-thirds had a monthly income of $\geq \$ 2000$. In terms of baseline HIV risk by study criteria, most participants $(72 \%)$ reported CAI with $\geq 3$ HIV-positive or unknown status partners in the last 3 months, and nearly half ( $46 \%$ ) reported being in a serodiscordant relationship (Table 1). Of note, there was no significant difference in the demographic and HIV risk profile between study completers and the initial TAPIR cohort.

\section{Baseline HIV risk category and partnership type}

The majority of participants were classified as having at least moderate HIV risk, with $27 \%$ of participants classified as high/very high, $29 \%$ moderate, and $44 \%$ low risk. In terms of partnership type at baseline, most participants reported multiple partners $(86 \%)$ with more than half having $>5$ partners. Of those with no or single partners, $n=50(13 \%)$ reported having one HIV-positive partner and only five participants (1\%) had no or one HIV-negative partner (Table 2).

\section{Change in HIV risk category and partnership type over time}

Of the 313 participants who reached week 48, there was no significant shift in $\mathrm{mCalcR}$ compared with baseline risk category, suggesting overall stability of recent HIV risk $(p=0.246$; Table 3$)$. However, there was a statistically significant change in partnership type from baseline to week 48, with an increase in the no/single- group and a corresponding decrease in the single+ and multi groups $(p<0.001$; Table 4$)$.

\section{PrEP adherence by HIV risk category and partnership type}

At week 48, HIV risk category as determined by mCalcR was found to be associated with TFV-DP levels (Kruskal-

Table 2. HIV Risk Score Category and Partnership Type

\begin{tabular}{|c|c|c|c|}
\hline & Week $0(\mathrm{n}=396)$ & Week $24(\mathrm{n}=349)$ & Week $48(\mathrm{n}=313)$ \\
\hline \multicolumn{4}{|l|}{ mCalcR category } \\
\hline Low $(<0.12)$ & $176(44 \%)$ & $146(42 \%)$ & $130(42 \%)$ \\
\hline Moderate $(0.12-0.59)$ & $115(29 \%)$ & $94(27 \%)$ & $76(24 \%)$ \\
\hline High/very high $(>0.59)$ & $105(27 \%)$ & $109(32 \%)$ & $107(35 \%)$ \\
\hline \multicolumn{4}{|l|}{ Partnership type } \\
\hline No or singe HIV-negative partner & $5(1 \%)$ & $33(9 \%)$ & $29(9 \%)$ \\
\hline Single HIV-positive partner & $50(13 \%)$ & $36(10 \%)$ & $29(9 \%)$ \\
\hline Multiple & $341(86 \%)$ & $280(80 \%)$ & $155(81 \%)$ \\
\hline $2-5$ partners & $127(32 \%)$ & $118(34 \%)$ & $97(31 \%)$ \\
\hline 5 partners & $214(54 \%)$ & $162(46 \%)$ & $158(50 \%)$ \\
\hline
\end{tabular}

mCalcR, modified Calculated Risk. 
Table 3. Change in HiV Risk Score Category Over Time

\begin{tabular}{lccccc}
\hline \multicolumn{5}{c}{ Week 48} \\
\cline { 2 - 5 } & Low & Moderate & High/very high & Total & McNemar's $\mathrm{p}$ \\
\hline Week 0 & & & & \\
Low & $76(24 \%)$ & $29(9 \%)$ & $34(11 \%)$ & $139(44 \%)$ & 0.246 \\
Moderate & $33(11 \%)$ & $28(9 \%)$ & $25(8 \%)$ & $86(27 \%)$ & \\
High/very high & $21(7 \%)$ & $19(6 \%)$ & $48(15 \%)$ & $88(28 \%)$ & \\
Total & $130(42 \%)$ & $76(24 \%)$ & $107(34 \%)$ & $313(100 \%)$ & \\
\hline
\end{tabular}

Wallis test; $p=0.018$ ). Participants in the moderate and high/very high risk mCalcR groups had higher adherence than the low-risk group with median TFV-DP levels of 1247 (IQR 988-1511) fmol/punch for moderate, 1186 (IQR 9711531) fmol/punch for high/very high, and 1026 (IQR 7181449 ) fmol/punch for low ( $p=0.027$ for high/very high vs. low; $p=0.012$ for moderate vs. low; Fig. 2a). Likewise, partnership type was associated with week 48 TFV-DP levels (Kruskal-Wallis; $p=0.007$ ). The 29 participants with no/single HIV-negative partner had lower adherence than those with either single HIV-positive partner or multiple partners with median TFV-DP levels of 835 (IQR 529-1207) fmol/punch for no/single-, 1099 (IQR 945-1427) fmol/punch for single+, and 1210 (IQR 885-1530) fmol/punch for multi ( $p=0.027$ single+ vs. no/single-; $p=0.002$ multi vs. no/single-; Fig. 2b). These effects were not observed for early study adherence at week 12 using baseline HIV risk category and partnership type.

\section{Discussion}

In a cohort of PrEP-taking MSM and TGW, partnership status changed over the course of the study with individuals shifting toward lower-risk partnerships, but objective HIV risk as calculated using mCalcR remained stable over 48 weeks. At the end of the study, individuals with higher HIV acquisition risk showed correspondingly higher levels of PrEP adherence.

While it is expected that relationships change over time, it was somewhat surprising that there was a shift toward lowerrisk partnerships over the study period. However, our study criteria ensured enrolling individuals at high risk for HIV acquisition, and thus there were few individuals with lowerrisk partnership types at baseline. Participants were enrolled in a PrEP demonstration study at a time of known risk as reflected in higher-risk partnerships. Over the 48 -week study period, relationships naturally changed, but perhaps we simply witnessed regression toward lower-risk HIV partnerships. To our knowledge, this finding is a unique observation as no other PrEP demonstration studies have specifically evaluated changes in partnership type.

Despite this increase in lower-risk partnerships, objective HIV risk based on the number of condomless anal sex acts and presence of an STI was constant throughout the study. Consistent with other open-label PrEP demonstration studies, eligibility criteria usually screen out low-risk persons, resulting in more individuals with recent risky behaviors. ${ }^{17}$ Current high-risk sexual behavior likely prompted these individuals to seek PrEP but also may have stabilized before starting a PrEP study. Moreover, participants were regularly reporting their risky sexual behaviors and simultaneously receiving risk reduction counseling, which may have led to increased self-monitoring and insight into the level of HIV risk.

In univariate analyses, both objective HIV risk and partnership type were associated with PrEP drug levels at week 48. Similar to findings from other clinical trials and demonstration projects, ${ }^{7-9}$ individuals engaging in higher-risk sexual behaviors showed durable adherence to PrEP compared with those with lower-risk behaviors. This finding implies that individuals may have recognized their HIV risk and acted to ensure protection from PrEP through attention to medication adherence. Conversely, individuals with lower PrEP adherence corresponded to lower-risk sexual behaviors, a logical reaction to reduced risk of HIV acquisition. ${ }^{18}$ As a result of regular monitoring and discussion of HIV risk behaviors and adherence during the study, individuals may have been able to appropriately titrate PrEP adherence to the level of risk. Likewise, MSM reporting higher-risk sexual partnership types (i.e., serodiscordant or multiple partners) were more adherent to PrEP than those in lower-risk partnerships. Although not addressed in this analysis, partnership dynamics beyond conferred HIV risk, including sex stigma, ${ }^{19}$ sexual health goals, ${ }^{20}$ and intimacy motivations,${ }^{21}$ have been shown

Table 4. Change in Partnership Type Over Time

\begin{tabular}{lccccc}
\hline \multicolumn{5}{c}{ Week 48} \\
\hline & No/single- & Single+ & Multi & Total & McNemar's $\mathrm{p}$ \\
\hline Week 0 & & & & & \\
No/single- & $0(0 \%)$ & $1(0.25 \%)$ & $1(0.25 \%)$ & $2(0.5 \%)$ & $<0.001$ \\
Single+ & $2(0.5 \%)$ & $22(6.75 \%)$ & $12(4.25 \%)$ & $36(11.5 \%)$ & \\
Multi & $27(8.5 \%)$ & $6(2 \%)$ & $242(77.5 \%)$ & $275(88 \%)$ & \\
Total & $29(9 \%)$ & $29(9 \%)$ & $255(82 \%)$ & $313(100 \%)$ & \\
\hline
\end{tabular}

No/single-, no partner/single HIV-negative partner; Single+, single HIV-positive partner; Multi, multiple partners:. 
a
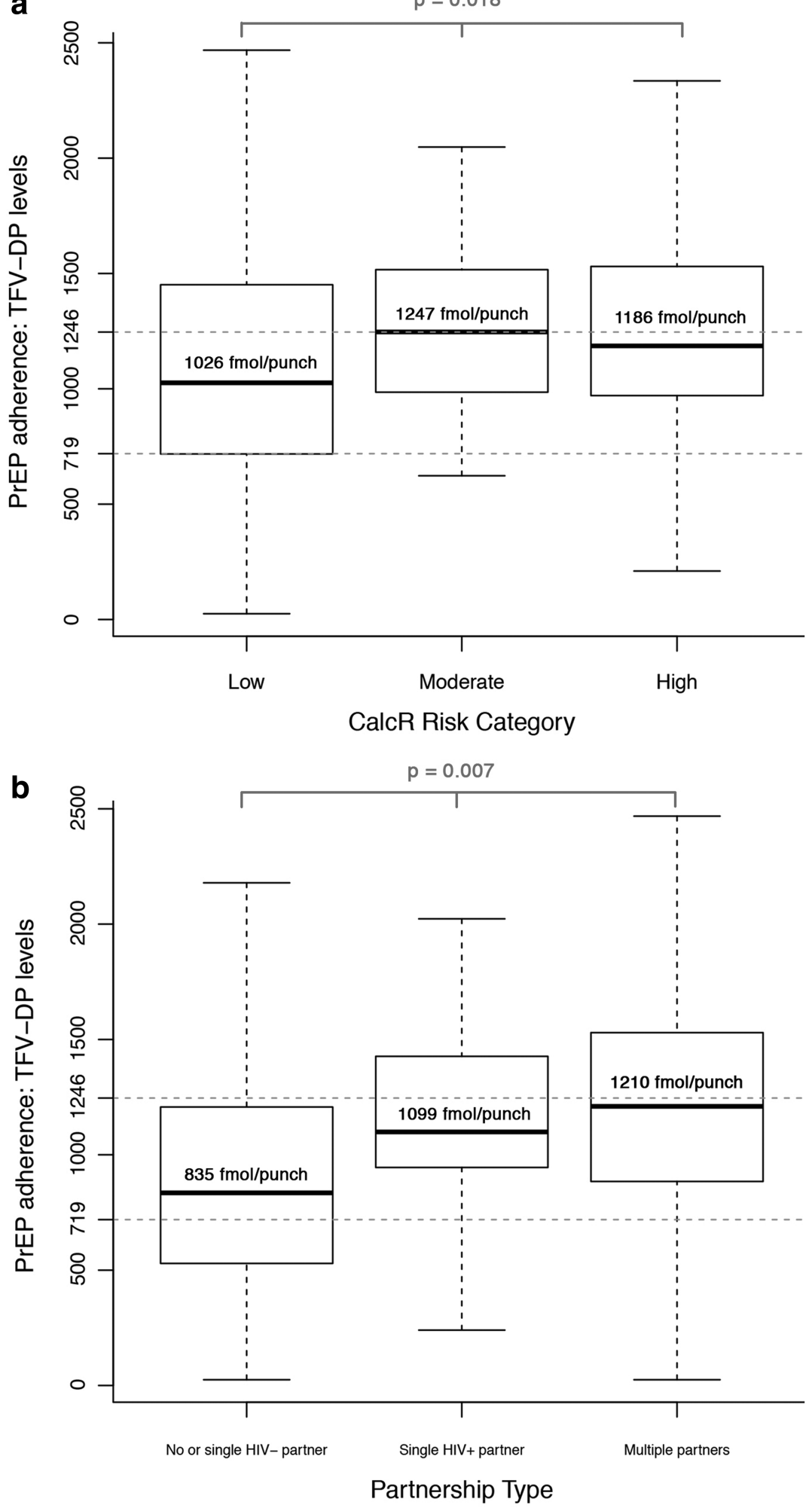

FIG. 2. The bottom and top of the boxes are the 25th percentile (Q1) and 75th percentile (Q3), respectively. The dark line in the box is the median TVF-DP level. The lower and upper whiskers represent the minimum and maximum values that do not exceed 1.5 times the interquartile range from the box. The brackets show the association between (a) HIV risk category and (b) partnership type with TFV-DP levels. The dotted lines represent adherence thresholds as defined in PK modeling studies by the lower interquartile value of protective (719 fmol/punch) and perfect (1246 fmol/punch) concentrations of TFVDP (Castillo-Mancilla JR, 2013). CalcR, Calculated Risk; PrEP, pre-exposure prophylaxis; TFV-DP, tenofovir-diphosphate. to impact PrEP use and adherence among MSM and may vary by age, race/ethnicity, ${ }^{22}$ and other factors. Further research exploring the interplay between relationship dynamics and sociodemographics on PrEP engagement and adherence is warranted.

Since sexual risk is not fixed over time, we hypothesized that HIV risk behavior/awareness and corresponding PrEP adherence would have a contemporaneous relationship. Indeed, we found no such association between HIV acquisition risk at study entry and PrEP adherence at 12 weeks, suggesting that current risk behaviors cannot be used to predict future adherence behavior. However, a recent study of MSM and TGW taking PrEP in Brazil showed an association between risk and consequent adherence where drug levels at 4 
weeks were higher among those reporting high-risk behavior at baseline. ${ }^{23}$ Still, the time between transmission and prevention behavior is relatively short, which reinforces the importance of temporal alignment of risk and adherence. ${ }^{18}$

In addition, the TAPIR study design may have inadvertently contributed to lower TFV-DP levels from individuals with former risk. Participants were expected to continue on PrEP for 48 weeks as study protocol prohibited restarting PrEP if it was stopped. Thus, for individuals where the reason for seeking PrEP was no longer relevant and HIV risk had dissipated, there would be no need to take PrEP consistently if at all while still remaining in the study. Needless to say, there will be times where PrEP may not be needed depending on perceived and/or actual risk. ${ }^{24}$ In real-world PrEP use, patients may reduce or stop PrEP use when a relationship has ended or they are not currently sexually active, and increase or restart PrEP if their risk behavior changes.

While our study had several strengths, including a large sample size, assessments at multiple time points, and a biologically measured adherence outcome, there are some limitations. First, the study enrollment criteria required that participants have persistent elevated risk of HIV acquisition. Thus, we had had very few individuals with low-risk partnership types and less than half with lower-risk behaviors at baseline. As stated above, the TAPIR study protocol did not allow participants to stop and restart PrEP during the study. Consequently, behaviors that qualified individuals for the study could have temporarily changed or entirely stopped, but they remained in study to maintain access to PrEP in the event HIV risk returned.

Additionally, similar to other established HIV risk calculators for MSM - such as the Centers for Disease Control and Prevention's HIV Incidence Risk Index for $\mathrm{MSM}^{25}$ and the San Diego Early Test Score ${ }^{26}$-mCalcR does not adjust for the viral load of HIV-infected partners and, as a result, may overestimate actual risk for individuals who have HIVpositive partners with suppressed HIV viral loads. Assessing the virological status of HIV-positive partners is difficult in the context of a research study since it either requires concurrent enrollment of partners for viral load measurement or relies on participants' accurate knowledge of partner virological status as we did in this study. ${ }^{27}$ Nearly $90 \%$ of participants with a single HIV-positive partner reported that their partner was virally suppressed. However, studies have found that primary partners tend to overestimate partner's viral load status. ${ }^{28}$ Revising the ranges of risk probability with and without knowledge of partner's virologic suppression may be a strategy to improve the validity of mCalcR.

Despite the overall high risk reported in our study, few individuals had very high $\mathrm{mCalcR}$ risk scores, which resulted in the combination of the two highest risk categories and potentially some loss of granularity in our findings. Our inability to meaningfully distinguish those with greatest HIV risk and the impact that degree of risk could have on prevention behaviors, including PrEP adherence, may imply the need to lower the higher mCalcR cut-points to identify, acknowledge, and ultimately support individuals with disproportionate risk of HIV acquisition. Our objective calculated HIV risk score and categories have not been evaluated in prospective studies of HIV incidence. While published and validated risk scores exist, ${ }^{25,26,29}$ the data and time frames of sex acts and STIs collected in TAPIR did not allow for the calculation of other HIV risk scores. In addition, the mathematical equation of the mCalcR score intentionally excludes sociodemographic risk factors as it was designed to focus explicitly on participant-specific biological transmission events.

The knowledge gained from this study may help clinicians know what to expect when putting individuals on PrEP. If our findings are generalizable to broader PrEP implementation, we might observe a shift toward lower risk partnerships in those who report higher-risk partnerships at PrEP initiation, but they continue to have sexual risk behaviors that are relatively stable over time. Moreover, at least in a population of MSM seeking PrEP, higher HIV risk behaviors may be associated with better PrEP adherence. In clinical practice, providers can reasonably assume that HIV risk and partnership status of MSM patients may change. Our findings highlight the importance of discussing HIV prevention strategies and offering PrEP when HIV risk is elevated, with the assurance that adherence should be sufficient for protection against HIV.

\section{Acknowledgments}

The authors would like to thank our participants for volunteering for this study. We would also like to acknowledge the efforts of our excellent study staff across all the CCTG. This work was supported by the California HIV Research Program (MC08-SD-700 and EI-11-SD-005). Additional funding includes NIH grants: AI 064086 (K24 to R.H.); AI 36214 (CFAR Clinical Investigation and Biostatistics Core); TR001444 (KL2 to J.B.). Portions of this work were previously presented at the HIV Research for Prevention Conference 2016 (R4P). Study drug was provided by Gilead Sciences and, DBS drug concentrations were paid for through a grant by Gilead Sciences.

\section{Author Disclosure Statement}

R.H. is Professor Emeritus at UCSD and a current employee of Gilead Sciences. The other authors do not have any conflicts of interest to report.

\section{References}

1. CDC. HIV in the United States: At A Glance. 2018; Available at: www.cdc.gov/hiv/statistics/overview/ataglance .html

2. Grant RM, Lama JR, Anderson PL, et al. Preexposure chemoprophylaxis for HIV prevention in men who have sex with men. N Engl J Med 2010;363:2587-2599.

3. Baeten JM, Donnell D, Ndase P, et al. Antiretroviral prophylaxis for HIV prevention in heterosexual men and women. N Engl J Med 2012;367:399-410.

4. Thigpen MC, Kebaabetswe PM, Paxton LA, et al. Antiretroviral preexposure prophylaxis for heterosexual HIV transmission in Botswana. N Engl J Med 2012;367:423434.

5. Grant RM, Anderson PL, McMahan V, et al. Uptake of preexposure prophylaxis, sexual practices, and HIV incidence in men and transgender women who have sex with men: A cohort study. Lancet Infect Dis 2014;14:820-829.

6. Grant RM, Glidden DV. HIV moments and pre-exposure prophylaxis. Lancet 2016;387:1507-1508. 
7. Liu AY, Cohen SE, Vittinghoff E, et al. Preexposure prophylaxis for HIV infection integrated with municipal- and community-based sexual health services. JAMA Intern Med 2016;176:75-84.

8. Liu A, Glidden DV, Anderson PL, et al. Patterns and correlates of PrEP drug detection among MSM and transgender women in the Global iPrEx Study. J Acquir Immune Defic Syndr 2014;67:528-537.

9. Donnell D, Baeten JM, Bumpus NN, et al. HIV protective efficacy and correlates of tenofovir blood concentrations in a clinical trial of PrEP for HIV prevention. J Acquir Immune Defic Syndr 2014;66:340-348.

10. Moore DJ, Jain S, Dube MP, et al. Randomized controlled trial of daily text messages to support adherence to PrEP in at-risk for HIV individuals: The TAPIR study. Clin Infect Dis 2018;66:1566-1572.

11. Blumenthal J, Jain S, Mulvihill E, et al. Perceived versus calculated HIV risk: Implications for pre-exposure prophylaxis uptake in a randomized trial of men who have sex with men. J Acquir Immune Defic Syndr 2019;80:e23-e29.

12. Milam J, Morris S, Jain S, et al. Randomized controlled trial of an internet application to reduce HIV transmission behavior among HIV infected men who have sex with men. AIDS Behav 2016;20:1173-1181.

13. Baggaley RF, White RG, Boily MC. HIV transmission risk through anal intercourse: Systematic review, meta-analysis and implications for HIV prevention. Int J Epidemiol 2010; 39:1048-1063.

14. Rottingen JA, Cameron DW, Garnett GP. A systematic review of the epidemiologic interactions between classic sexually transmitted diseases and HIV: How much really is known? Sex Transm Dis 2001;28:579-597.

15. Centers for Disease Control and Prevention. HIV among Gay and Bisexual Men. Available at: www.cdc.gov/nchhstp/ newsroom/docs/factsheets/cdc-msm-508.pdf (Last accessed June 10, 2018).

16. Castillo-Mancilla JR, Zheng JH, Rower JE, et al. Tenofovir, emtricitabine, and tenofovir diphosphate in dried blood spots for determining recent and cumulative drug exposure. AIDS Res Hum Retroviruses 2013;29:384-390.

17. Jenness SM, Sharma A, Goodreau SM, et al. Individual HIV risk versus population impact of risk compensation after HIV preexposure prophylaxis initiation among men who have sex with men. PLoS One 2017;12:e0169484.

18. Haberer JE, Kidoguchi L, Heffron R, et al. Alignment of adherence and risk for HIV acquisition in a demonstration project of pre-exposure prophylaxis among HIV serodiscordant couples in Kenya and Uganda: A prospective analysis of prevention-effective adherence. J Int AIDS Soc 2017;20:21842.

19. Grace D, Jollimore J, MacPherson P, Strang MJP, Tan DHS. The pre-exposure prophylaxis-stigma paradox: Learning from Canada's first wave of PrEP users. AIDS Patient Care STDs 2018;32:24-30.

20. Gamarel KE, Hilley A, Boonrai K, Radix A, Golub SA. Relationship Dynamics and Pre-Exposure Prophylaxis (PrEP) Adherence Self-Efficacy Among Men Who have Sex with Men (MSM) in Romantic Relationships. Chicago, IL: Paper presented at: American Public Health As- sociation Annual Meeting, Oct 31-Nov 4, 2015, Abstract 326952.

21. Gamarel KE, Golub SA. Intimacy motivations and preexposure prophylaxis (PrEP) adoption intentions among HIV-negative men who have sex with men (MSM) in romantic relationships. Ann Behav Med 2015;49:177-186.

22. Tan JY, Campbell CK, Conroy AA, Tabrisky AP, Kegeles S, Dworkin SL. Couple-level dynamics and multilevel challenges among black men who have sex with men: A framework of dyadic HIV care. AIDS Patient Care STDs 2018;32:459-467.

23. Hoagland B, Moreira RI, De Boni RB, et al. High preexposure prophylaxis uptake and early adherence among men who have sex with men and transgender women at risk for HIV Infection: The PrEP Brasil demonstration project. J Int AIDS Soc 2017;20:21472.

24. Amico KR, Stirratt MJ. Adherence to preexposure prophylaxis: Current, emerging, and anticipated bases of evidence. Clin Infect Dis 2014;59 Suppl 1:S55-S60.

25. Smith DK, Pals SL, Herbst JH, Shinde S, Carey JW. Development of a clinical screening index predictive of incident HIV infection among men who have sex with men in the United States. J Acquir Immune Defic Syndr 2012;60: 421-427.

26. Hoenigl M, Weibel N, Mehta SR, et al. Development and validation of the San Diego Early Test Score to predict acute and early HIV infection risk in men who have sex with men. Clin Infect Dis 2015;61:468-475.

27. Morris S, Jain S, Kalvo K, et al. Comparison of Behavioral Factors in Men Who Have Sex with Men who go on PrEP by Partner Status. Arlington, VA: Paper presented at: National Summit on HCV and HIV Diagnosis, Prevention and Access to Care. June 4-6, 2015, Abstract 68.

28. Stolte IG, de Wit JB, van Eeden A, Coutinho RA, Dukers NH. Perceived viral load, but not actual HIV-1-RNA load, is associated with sexual risk behaviour among HIVinfected homosexual men. AIDS 2004;18:1943-1949.

29. Hyman S, Vittinghoff E, Irving R, et al. Sex Pro: A Personalized HIV Risk Assessment Tool for Men Who Have Sex With Men. Seattle, WA: Conference on Retrovirusus and Opportunistic Infections; February 23-26, 2015, Abstract 1017.

Address correspondence to: Jill Blumenthal, $M D$

Department of Medicine

University of California San Diego (UCSD)

220 Dickinson Street Suite A San Diego, CA 92103

E-mail: jblumenthal@ucsd.edu

Sheldon R. Morris, MD, MPH Department of Medicine

University of California San Diego (UCSD)

220 Dickinson Street Suite A San Diego, CA 92103

E-mail: shmorris@ucsd.edu 